\title{
基于等效风场试验的QTT台址风场分布调控技术 方案初探
}

王从思 ${ }^{1,2^{*}}$ ，王雪晴 ${ }^{1}$, 许谦 ${ }^{2 *}$, 王娜 ${ }^{2}$, 郑元鹏 ${ }^{3}$, 杜彪 ${ }^{3}$, 连培园 ${ }^{1}$, 薛松 ${ }^{1}$, 严粤飞 ${ }^{1}$, 段玉虎 ${ }^{4}$, 石禹 ${ }^{5}$, 贾昱 ${ }^{5}$

1. 西安电子科技大学电子装备结构设计教育部重点实验室, 西安 710071;

2. 中国科学院新疆天文台, 乌鲁木齐 830011 ;

3. 中国电子科技集团公司第五十四研究所, 石家庄 050081 ;

4. 中国电子科技集团公司第三十九研究所, 西安 710065 ;

5. Department of Mechanical Engineering, University of Chester, Chester CH1 4BJ, UK

*联系人, 王从思, E-mail: congsiwang@163.com; 许谦, E-mail: xuqian@xao.ac.cn

收稿日期: 2018-12-31; 接受日期: 2019-03-04; 网络出版日期: 2019-06-11

国家重点基础研究发展计划(编号: 2015CB857100)、陕西省自然科学基金(编号: 2018JZ5001)、天山创新团队计划(编号: 2018D14008)、国家 自然科学基金(编号: U1737211)和中国科学院西部之光计划(编号: 2017-XBQNXZ-B-024)资助项目

摘要 针对高频段大口径全向可动射电望远镜QTT对电性能的高要求, 必须降低风扰动对天线的影响. 本文分析 了QTT台址地形地貌以及风场分布的特点，提出了一种主动降低天线所在区域风速的QTT台址风场分布调控技 术方案，进一步搭建了用于QTT台址风场分布调控微缩模型的等效风场试验，通过试验数据分析，确定了QTT风 场分布调控技术方案的可行性, 并初步给出了经风场调控后, 风速显著降低的区域范围以及风速降低的幅度, 为 QTT台址风场分布的调控提供了技术依据，从而可大大增加射电望远镜的有效观测时间.

关键词射电望远镜, 反射面天线, 风场分布, 风场调控

PACS: 95.55.Jz, 84.40.Ba, 92.60.Fm, 89.20.Bb

\section{1 引言}

伴随着人类历史的发展和科学技术的进步，射电 望远镜观测技术的发展, 极大推动了天文学的发展, 成为其发展的主要动力之一. 望远镜结构的反射面面 积是射电望远镜性能的重要指标之一，大口径的射电 望远镜可以观测更多、更远的天体 ${ }^{[1,2]}$. 世界上目前已
建成的最大口径全可动射电望远镜是美国于2000年建 成的绿岸射电望远镜 $(\mathrm{GBT}$ ，口径 $100 \times 110 \mathrm{~m}$ ). 我国目 前最大口径全可动射电望远镜为上海天文台的 $65 \mathrm{~m}$ 口 径射电望远镜, 并拟在新疆奇台县建设 $110 \mathrm{~m}$ 口径全可 动射电望远镜 $(\mathrm{QTT})$.

为满足国际天文学发展需求, 大口径全可动射电 望远镜的研究及建设问题已成为当前天文学领域关注

引用格式: 王从思, 王雪晴, 许谦, 等. 基于等效风场试验的QTT台址风场分布调控技术方案初探. 中国科学: 物理学 力学 天文学, 2019, 49: 099515 Wang C S, Wang X Q, Xu Q, et al. Preliminary study of regulation technology of wind field distribution on QTT site based on test of equivalent wind field (in Chinese). Sci Sin-Phys Mech Astron, 2019, 49: 099515, doi: 10.1360/SSPMA2018-00422 
的热点之一. 对于巨型射电望远镜结构, 由于其反射面 面积巨大，重力、风扰、温差等因素会引起望远镜反 射面的形状变化 ${ }^{[3-6]}$, 降低反射面的形状拟合精度, 引 起偏焦和指向误差, 降低望远镜的灵敏度及分辨率, 影 响其工作性能, 其中重力、温差等为慢变环境因素, 所 产生的结构变形对天线指向精度的影响可通过提前对 其估算并建立表格进行补偿, 而风扰动则由于其随机 性和时变性，已成天线观测环境载荷中最复杂也是最 难以解决的问题 ${ }^{[7,8]}$.

在以往，大多数天线都是通过三种方法来减少风 扰动对天线性能的影响 ${ }^{[9-11]}$. (1) 采用闭环控制系统实 现指向控制，其实质是根据指向要求方位和俯仰方向 分别控制其转轴旋转到指定位置，转角位置由多极旋 转变压器或高精度编码器检测. 为提高跟踪性能和可 靠性，目前普遍采用基于PID的位置环、速度环和电 流环三闭环控制方式 ${ }^{[12,13]}$. 但这种方法对于大口径、 高频段、高指向精度的反射面天线而言，已不能满足 要求; (2) 从鲁棒控制和最优控制理论出发, 提出了相 应的抗风载控制补偿方法, 但据此提出的种种补偿方 法, 其补偿能力均非常有限; (3) 在天线外部修建一个 天线罩来隔绝外部环境因素对天线的影响 ${ }^{[14]}$, 虽然天 线罩对减小风荷载有很明显的作用，但天线罩是天线 前面的障碍物，对天线辐射波会产生吸收和反射，改 变天线的自由空间能量分布 ${ }^{[15]}$, 并在一定程度上影响 天线性能. 而且天线罩大大增加了工程造价, 对大型 天线来说是不现实的.

为此，本文提出了一种QTT台址风场分布调控技 术，与原有的被动地对天线进行抗风扰控制和补偿不 同，该技术方案思路为通过研究台址地形地貌特点、 风场分布规律, 在台址区域内合理地布置林带、林网, 来调控台址的风场分布，可以降低天线所在区域风场 的风速，减少系统对天线抗风扰控制和补偿的工作量, 增加天线在极限环境条件下的工作时长，从而主动地 减少风扰动对天线的影响，以增加射电望远镜的有效 观测时间.

\section{2 风场分布调控技术方案}

如图1所示, QTT台址距奇台县城以南直线距离约 $46 \mathrm{~km}$, 位于东天山北麓，是一处东西约 $1.5 \mathrm{~km}$ 、南北 约 $2 \mathrm{~km}$ 、四面环山的矩形盆地, 周围山梁海拔从

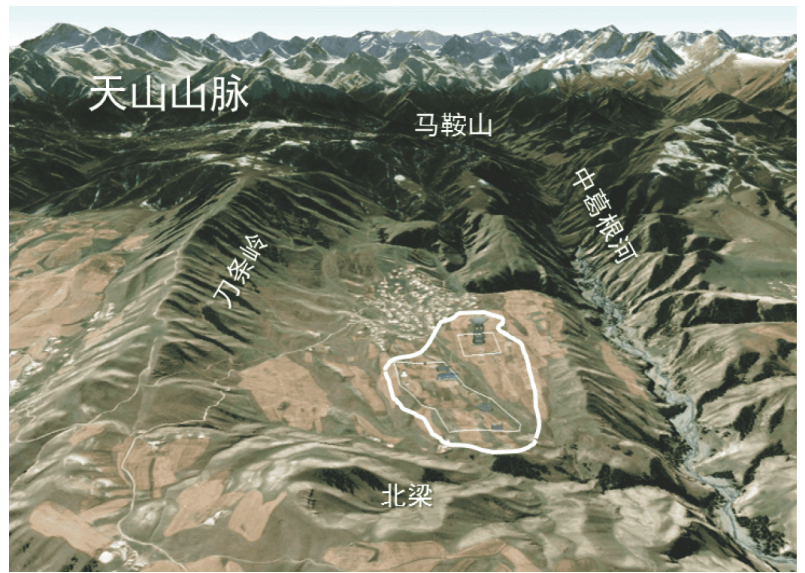

图 1 (网络版彩图)QTT台址示意图

Figure 1 (Color online) The site for QiTai Telescope.

1860-2250 m不等, 与周围区域形成较好的隔离, 盆地 内海拔约1730-1830 m. 其东北部和西南部山梁较高, 台址区域风向常年为自西北向东南方向，即图中自右 下到左上方向. 其地形地貌和环境风场条件适合通过 在台址区域内合理布置林带、林网来进行风场分布调 控, 如图2所示为QTT台址风场调控技术方案.

\section{1 建立环境风场的分布数据库}

根据已有的大气边界层风特性，天线台址处的地 形地貌特征以及天线台址处多年的风场观测资料，其 中包括在不同时刻下各观测位置处的风场情况，合理 简化QTT台址地形地貌、获知环境风场分布规律，建 立环境风场数据库.

\section{2 微缩模型等效风场试验方法}

风洞试验源自美国，起初是建立大气边界层风洞， 随后这项研究手段发展起来, 目前国内的风洞技术也 逐渐完备起来. 风洞试验是一种以空气动力学理论为 基础的试验方法，在模拟的风洞中安放要研究的实体 模型，一般试验模型是按照实物缩小一定的比例，给 模型一个相对速度值, 来研究气体流动对结构产生的 影响, 从试验获得数据, 来进一步来对结构进行分析 和计算. 由于风洞试验是在较稳定的室内环境中进行, 可避免由于天气等偶然因素对结果产生的偏差, 这也 使得对速度和温度的控制更加精准.

本方案根据风洞试验原理，拟设计等效风场试验， 设计搭建台址地理环境的微缩模型、用于风场分布调 


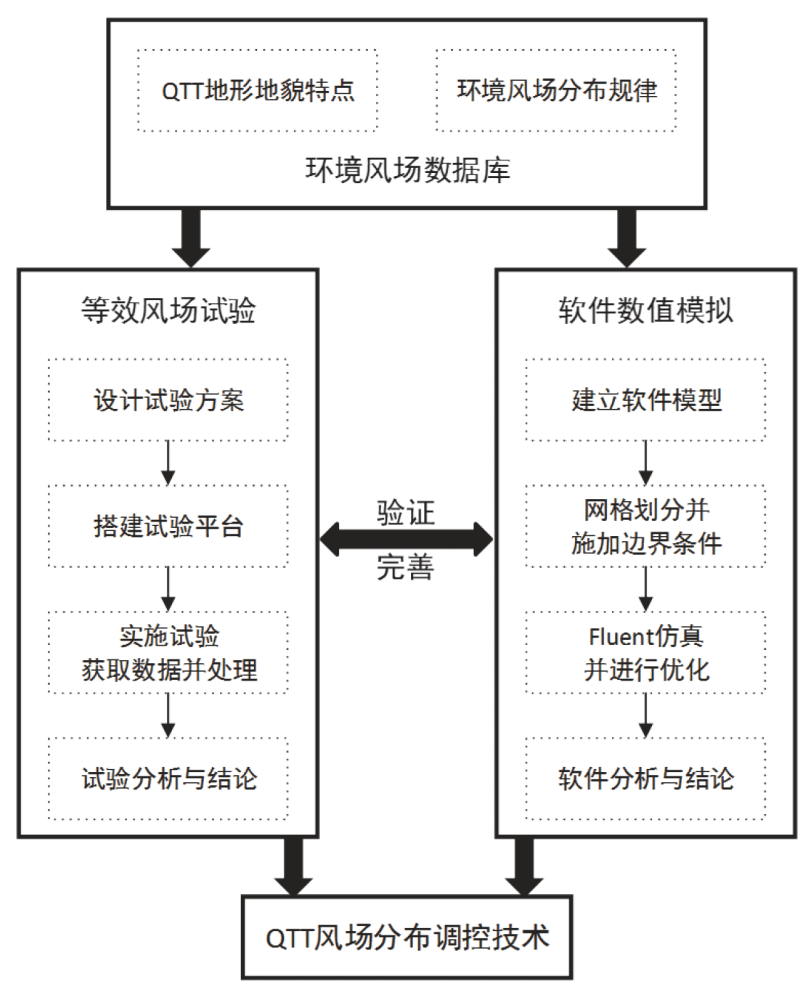

图 2 QTT台址风场分布调控技术方案

Figure 2 The regulation scheme for wind distribution.

控的林带的微缩模型以及射电望远镜的微缩模型，在 等效风洞中，对整体模型进行等效风场试验，探索望 远镜台址的风场分布调节规律.

风洞试验方法虽然已经日渐成熟，但仍存在一定 的局限性. (1) 由于模型是按一定比例做成的微缩模 型, 所以对于细微处和突变处流动特性的描述存在弊 端，而且实物上较小结构处的流场情况信息较难获取; (2) 由于自然界中大多数实际工程情况, 都将风作为大 气边界层内的近地风探讨，模拟的流场对于大气边界 层的风的流动会有差别, 也会对结果有影响; (3) 微缩 模型的制作很有考究，从开始就要精致周到的设计好 各种条件, 模型的制作成本较高; (4) 风洞试验不能模 拟强风，对一定因素下出现的罕遇情况下风场变化的 分析无能为力, 得不到强风的作用效应, 就无法开展 相应的研究.

\section{3 计算机数值模拟方法}

实测方法的缺陷和风洞试验的限制，从一定程度 上推进了计算风工程的发展. 计算机数值模拟方法避
开了上述问题，该法以计算流体力学为基础，借助于 先进的计算机技术，能得到更准确全面的数据，还可 以通过更加直观的图形来观察变化趋势，这种方法既 节省人力, 经济投入也不会太大, 可行性大, 并且突出 的优势是可以模拟多种情况下的风载效应，如风速改 变、尺寸改变、材料改变、湍流模型改变等，这大大 降低了人员的工作量和资金投入，周期短，还可以得 到不同条件下的计算结果用以比较分析，信息完整性 和精确度更高，具有现实意义，因而渐渐被应用于实 践工程中.

本研究方案在等效风风场试验基础上进行计算机 数值模拟分析. 计算机数值模拟分析拟基于 GIS 数据 来拟合得出天线台址地形地貌曲面 ${ }^{[16]}$, 然后利用Fluent进行风场数值求解, 通过数值模拟结果与试验实测 结果进行对比分析、验证和完善风场调控技术.

\section{3 风场分布调控技术方案可行性分析}

为分析QTT台址风场分布调控技术方案的可行 性, 首先搭建了一个简单的等效风场试验平台, 进行了 微缩模型等效风场试验, 该试验平台如图3所示.

\section{1 搭建等效风场试验平台}

该试验平台基于相似理论设计，主要由等效风场 部分、风场调控板部分和风速测试装置部分三部分组 成. 等效风场部分用于模拟天线台址处的风场, 风场调

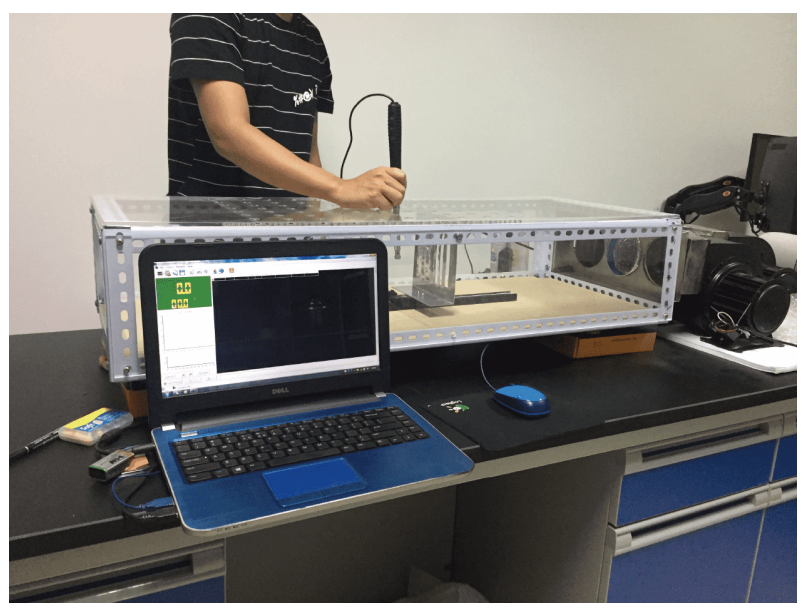

图 3 (网络版彩图)微缩模型等效风场试验平台

Figure 3 (Color online) The experimental facility for miniature model of antenna. 
控板部分是用于模拟林带对环境风场的调控作用，风 速测试装置则是用于对试验风场风速数据的获取. 该 平台基本上具备了本文所提调控技术方案的必须 条件.

等效风场部分由风道和风机组成，风道用来模拟 望远镜台址区域部分的风场, 结合试验目标和等效风 场试验条件，制作了缩尺比为 $1: 4000$ 的试验风道，其俯 视图如图4所示, 风道内风向从左至右, 其顶部开测试 孔以方便传感器伸入测试风速, 测试孔间距 $50 \mathrm{~mm}$, 呈 7 行 10 列阵列分布, 风机作为提供风道内所需的风场的 风源，如图3中最右侧部分所示，根据QTT台址区域的 风场特性资料，可按大气边界层的平均风速剖面的指 数率来描述, 即

$U(z)=U_{\mathrm{r}}\left(z / z_{\mathrm{r}}\right)^{\alpha}$,

其中 $z$ 为任意高度, $U(z)$ 为 $z$ 处的平均风速， $z_{\mathrm{r}}$ 为某一参 考高度, $U_{\mathrm{r}}$ 为参考高度处的平均风速, $\alpha$ 为地面粗糙度 指数, 其数值与地面粗粘度类别有关, QTT台址区域的 风场特性参数基本与 $\mathrm{B}$ 类场地风剖面参数相符，本试 验中风道内风场情况与其也基本相符.

风场调控板为铝板所制, 如图5所示, 风场调控板 1 为开了阵列孔洞的铝板, 风场调控板 2 为在其左右两侧 各开了 3 个约 $45^{\circ}$ 角导流缝的铝板, 以作为对环境风场 进行调控的林带的等效微缩模型. 试验时将其底部固 定在风道底板上，安装位置在测试孔第2列和第3列中 间，横跨3-5行，风场调控板俯视安装位置如图4中绿 色宽线所示, 在试验风道内的空间位置见图5, 这样可 以测量板前后的风速，且满足其前方风场改变区域 小、其后方风场改变区域大的合理性.

风速测试装置是型号为希玛AR866的热敏式风速 仪, 其主要参数如表 1 所示, 进行试验时如图3所示手持

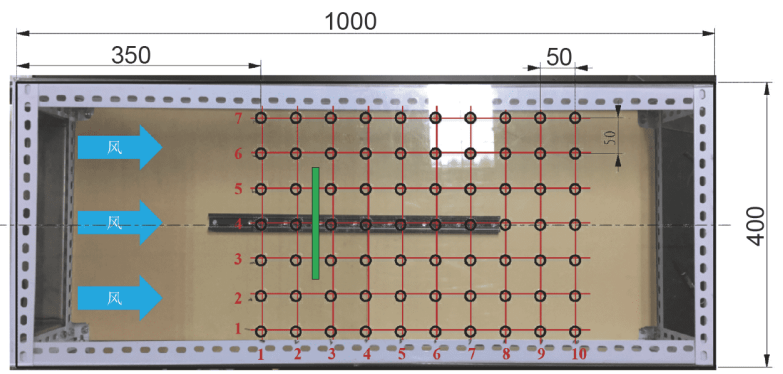

图 4 (网络版彩图)试验风道及测试孔俯视图

Figure 4 (Color online) The wind channel and test hole.

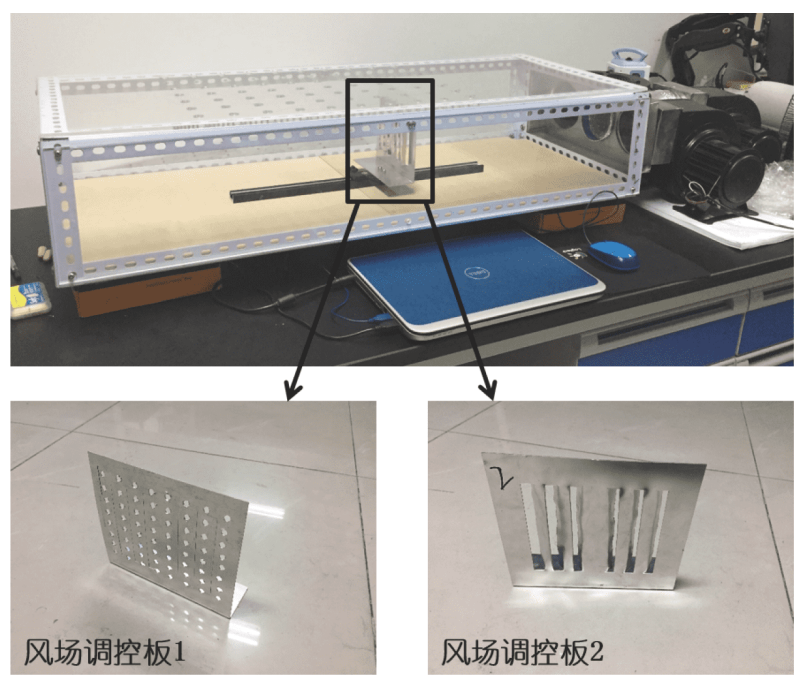

图 5 (网络版彩图)风场调控板

Figure 5 (Color online) Regulation plane with different holes and diversion slots.

表 1 AR866的主要性能参数表

Table 1 AR866 main performance parameter table

\begin{tabular}{cc}
\hline 技术指标 & AR866 \\
\hline 风速测量范围 & $0-30 \mathrm{~m} / \mathrm{s}$ \\
风速测量误差 & $\pm 1 \%$ \\
风温测量范围 & $0-45^{\circ} \mathrm{C}$ \\
温度误差 & $\pm 1{ }^{\circ} \mathrm{C}$ \\
风速分辨率 & $0.01 \mathrm{~m} / \mathrm{s}$ \\
USB外接电脑功能 & 有 \\
\hline
\end{tabular}

握杆坚直伸入如图4所示的测试孔中不同长度, 来使传 感器探头位于风场内的不同高度, 测得 70 个测试孔在 3 个高度，即距离试验风道底部 $125 ， 75$ 和 $25 \mathrm{~mm}$ 位置处 的风速，连接计算机，使用风速仪配套Anemometer计 算机软件记录数据，每个测试点测得并保存连续时间 内的 10 个风速数据, 导出Excel文件以便后期处理.

\section{2 试验风场测量与结果}

在本次等效风洞试验中，首先控制试验风道内的 风速，使其较符合台址真实风场环境，利用传感器测 试，得出未调控前的环境风场分布情况，然后安装风 场调控板改变试验风道内风场分布，再测试得出经风 场调控后的风场分布.

通过对不安装风场调控板的环境风场、使用风场 
表 2 试验数据处理方法表

Table 2 Experimental data processing method table

\begin{tabular}{ccc}
\hline 技术指标 & 属性1 & 属性2 \\
\hline 折线图 & 不同工况 & 同一高度 \\
侧剖云图 & 同一工况 & 不同高度 \\
\hline
\end{tabular}

调控板 1 的工况 1 和使用风场调控板 2 的工况 2 这 3 个情 况下在 3 个高度处的风速测试，共得到630个风场风速 数据. 我们对这些数据进行分析, 用测试点的 2 个属性 分 2 种方式来处理数据, 数据处理方法详见表 2 .

图6为风场分布折线图, 横坐标为风速测试点所在 的列数, 纵坐标为风速大小 $(\mathrm{m} / \mathrm{s})$, 高度 1 为距离试验风 道底部 $125 \mathrm{~mm}$ 的高度, 高度 2 为距离试验风道底部 $75 \mathrm{~mm}$ 的高度, 高度3为距离试验风道底部 $25 \mathrm{~mm}$ 的高 度，图例为蓝色三角形的线型代表环境风场风速，橙 色矩形的线型代表工况1风场风速，灰色 $\times$ 形的线型代 表工况2风场风速, 其中试验风道内的风向是向横坐
标正向，即每幅折线图表示在两种调控情况下同一高 度处、同一行、不同列的测试点的风速情况. 可以看 出, 在所有的 3 个高度以及 3 行的 9 个风速折线图中, 未 经调控的环境风场与调控后的工况 1 、工况2的风速有 较大差异, 在调控板前的1, 2两列风速差异很小, 在调 控板后的 3 至 10 列风速差异较大, 可明显看出风场风速 经调控后风速显著降低.

图7为风场分布侧剖面云图, 是使用实测数据经过 插值得到的风场风速云图. 横坐标为风速测试点所在 的列数, 纵坐标为测试点高度, 实测了高度 1 、高度 2 和高度 3 这 3 个高度, 右侧图例为各颜色所代表的风速 $(\mathrm{m} / \mathrm{s})$ 的大小，颜色越偏红代表风速越高，颜色越偏蓝 色代表风速越低，即每幅侧剖面云图表示的是同一调 控情况下不同高度同一行不同列的测试点风速情况. 可以看出未加调控时的环境风场是在较低高度上风速 较小, 较高高度上风速较大, 这是符合大气边界层平均 风速剖面的指数律的, 通过对工况 1 和工况 2 风场分布 侧剖面图的观察可以发现，经调控后风速显著降低的
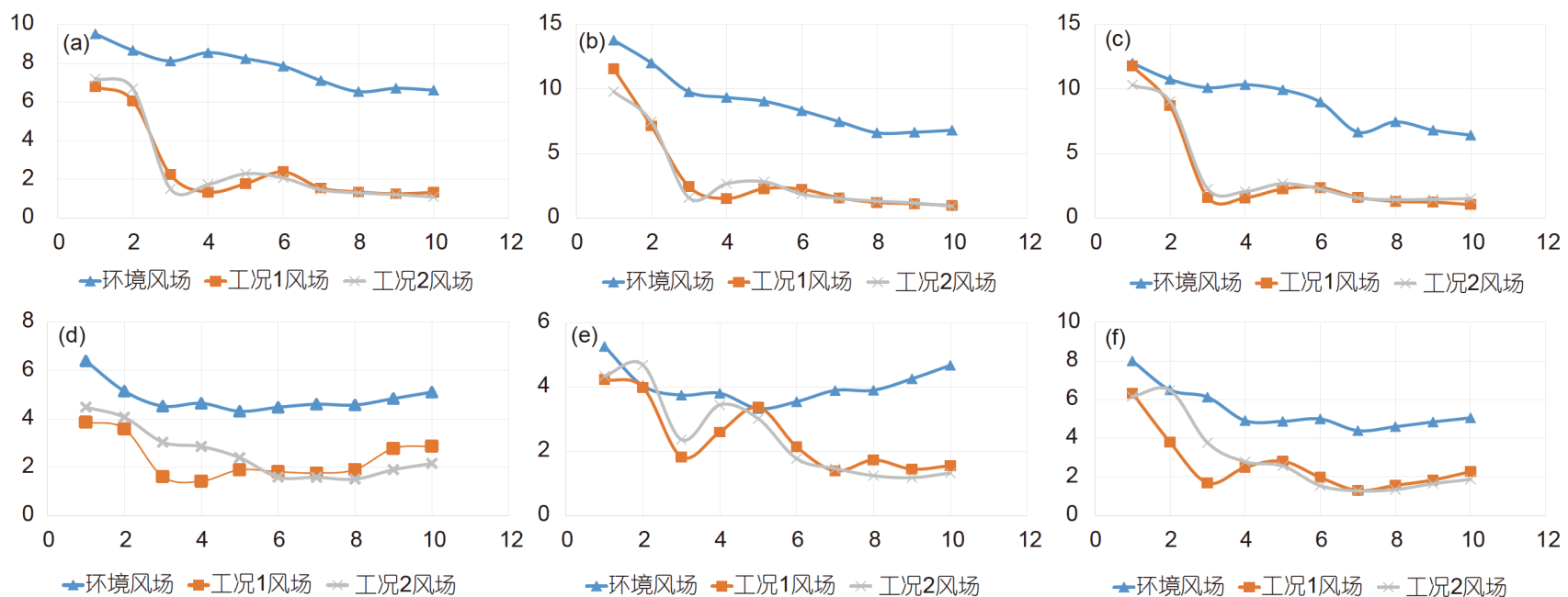

6

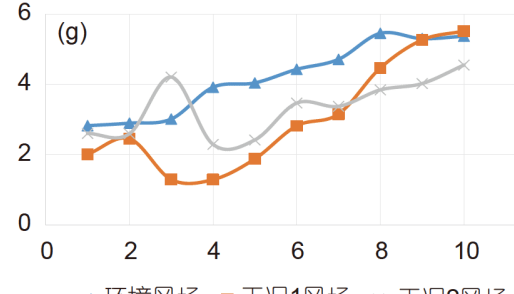

6 (e)

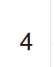

(e)

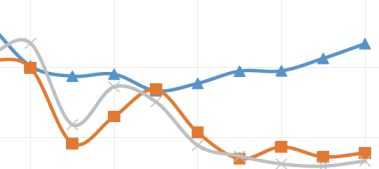

0

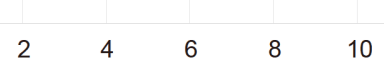

上环境风场 $=$ 工况1风场 $*$ 工况2风场

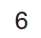

6 (h)

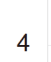

(h)

4

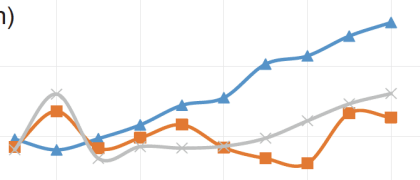

$12^{0}$

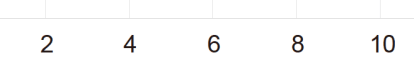

上环境风场

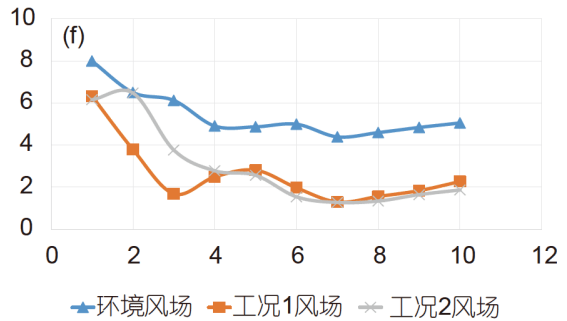

一坏境风场

\section{8}

6

(i)

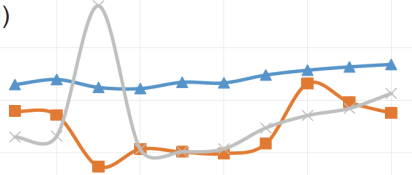

$\begin{array}{llllll}2 & 4 & 6 & 8 & 10 & 12\end{array}$ 上环境风场 $=$ 工况1风场

图 6 (网络版彩图)风场分布折线图. (a) 1高度第3行; (b) 1 高度第4行; (c) 1 高度第5行; (d) 2高度第3行; (e) 2 高度第4行; (f) 2 高 度第5行; (g) 3高度第3行; (h) 3高度第4行; (i) 3高度第5行

Figure 6 (Color online) The polygonal line map for wind speed distribution. (a) Height 1 line 3; (b) height 1 line 4; (c) height 1 line 5; (d) height 2 line 3; (e) height 2 line 4; (f) height 2 line 5; (g) height 3 line 3; (h) height 3 line 4; (i) height 3 line 5. 


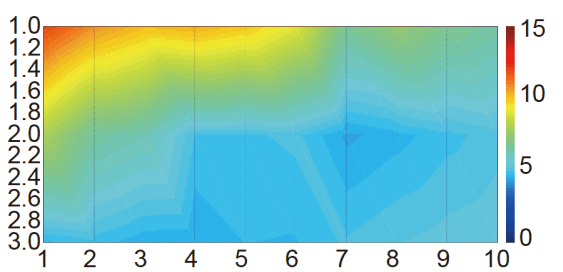

(a)

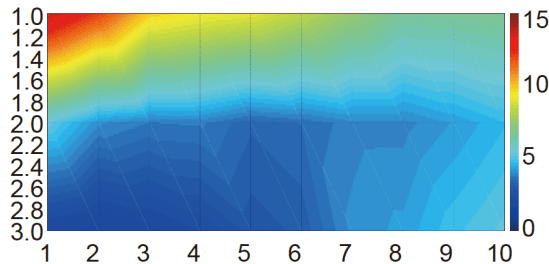

(d)

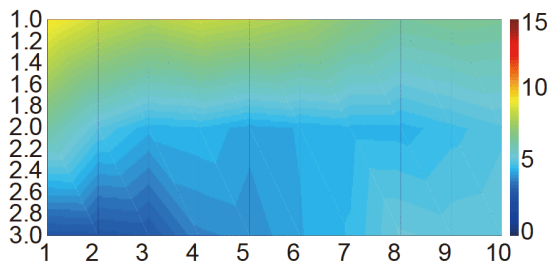

(g)

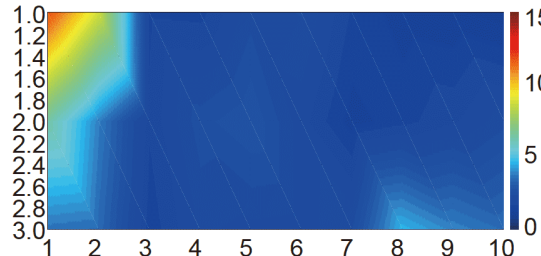

(b)

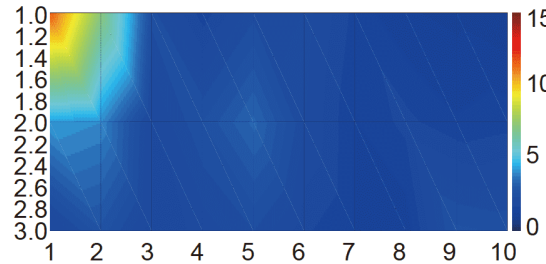

(e)

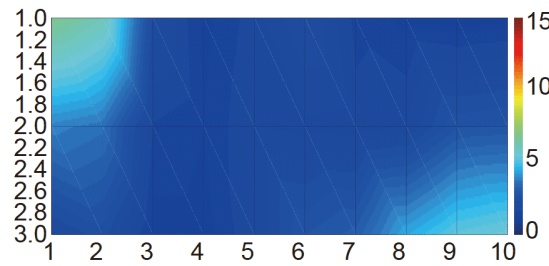

(h)

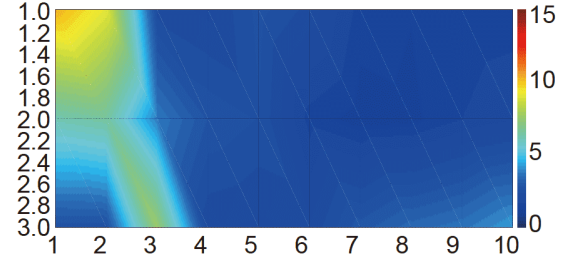

(c)

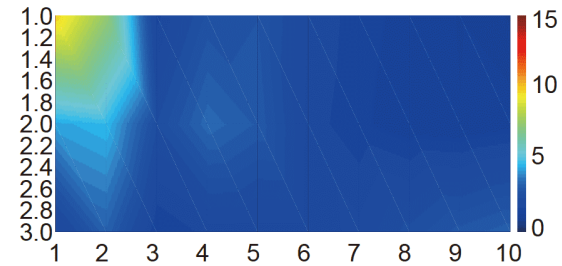

(f)

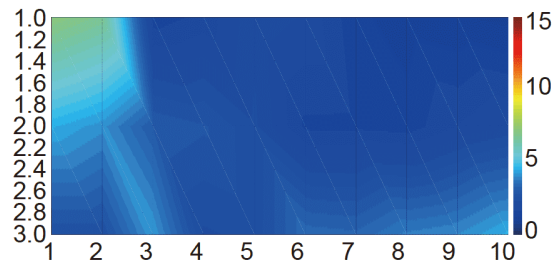

(i)

图 7 (网络版彩图)风场分布侧剖面云图. (a) 环境风场第5行; (b) 工况1风场第5行; (c) 工况2风场第5行; (d) 环境风场第4行; (e) 工况1风场第4行; (f) 工况2风场第4行; (g) 环境风场第3行; (h) 工况1风场第3行; (i) 工况2风场第3行

Figure 7 (Color online) The wind speed distribution nephogram in side view. (a) Environmental wind field line 5; (b) case 1 line 5; (c) case 2 line 5; (d) environmental wind field line 4; (e) case 1 line 4; (f) case 2 line 4; (g) environmental wind field line 3; (h) case 1 line 3; (i) case 2 line 3.

区域在高度1-2和第5-9列的这一区域范围内.

通过综合以上分析试验获得的数据，以及后处理 中得到的风场风速分布折线图和插值云图，可得出如 下结论：(1) 风场分布调控技术方案对改变风场情 况、降低区域风速确实有明显作用; (2) 风场调控板 对其后方风场的主要作用区域为3-5行、5-9列以及1 和2高度所围成的这一空间区域; (3) 从测得的数据中 可以看出，风场风速从原始的 $5-8 \mathrm{~m} / \mathrm{s}$ 左右，变为风场 调控板后方区域在 $1-2 \mathrm{~m} / \mathrm{s}$ 左右, 风速降低约 $75 \%$.

\section{4 结论}

本文针对QTT台址风场分布调控技术展开研究, 首先介绍了射电望远镜受风扰动影响的传统解决方 法, 然后根据QTT台址的地理位置和风场情况, 提出了 一种全新的主动地风场分布调控技术方案，并通过微 缩模型的等效风洞试验，确定了风场分布调控技术方 案的可行性，为进一步的计算机软件仿真、优化和试 验等方法的研究与实施提供依据.

\section{参考文献}

1 Rahmat-Samii Y, Haupt R. Reflector antenna developments: A perspective on the past, present and future. IEEE Antennas Propag Mag, 2015, 57: $85-95$

2 von Hoerner S. Design of large steerable antennas. Astron J, 1967, 72: 35

3 Greve A, Morris D. Repetitive radio reflector surface deformations. IEEE Trans Antennas Propagat, 2005, 53: $2123-2126$

4 Greve A, Karcher H J. Performance improvement of a flexible telescope through metrology and active control. Proc IEEE, 2009, 97: 1412-1420

5 Duan B Y, Wang C S. Reflector antenna distortion analysis using MEFCM. IEEE Trans Antennas Propagat, 2009, 57: 3409-3413

6 Wang C S, Duan B Y, Qiu Y Y. On distorted surface analysis and multidisciplinary structural optimization of large reflector antennas. Struct Multidisc Optim, 2007, 33: 519-528

7 Gawronski W. Control and pointing challenges of large antennas and telescopes. IEEE Trans Contr Syst Technol, 2007, 15: 276-289 
8 Wang C S, Xiao L, Xiang B B, et al. Development of active surface technology of large radio telescope antennas (in Chinese). Sci Sin-Phys Meth Astron, 2017, 47: 059503 [王从思, 肖岗, 项斌斌, 等. 大型射电望远镜天线主动面补偿研究进展. 中国科学: 物理学 力学 天文学, 2017, 47: 059503]

9 Gawronski W. Modeling wind-gust disturbances for the analysis of antenna pointing accuracy. IEEE Antennas Propagat Magazine 2004, 46: 50-58

10 Ukita N, Ezawa H, Ikenoue B, et al. Thermal and wind effects on the azimuth axis tilt of the ASTE 10-m antenna. Nobeyama Radio Observatory, 2007, 10: 25-33

11 Wang C S, Wang N, Lian P Y, et al. Thermal Deformation Compensation Technology For High Frequency Large Reflector Antenna (in Chinese). Beijing: Science Press, 2018 [王从思, 王娜, 连培园, 等. 高频段大型反射面天线热变形补偿技术. 北京: 科学出版社, 2018]

12 Zhang J, Huang J, Zhou J, et al. A compensator for large antennas based on pointing error estimation under a wind load. IEEE Trans Contr Syst Technol, 2017, 25: 1912-1920

13 Zhang J, Huang J, Wang S, et al. An active pointing compensator for large beam waveguide antenna under wind disturbance. IEEE/ASME Trans Mech, 2016, 21: 860-871

14 Wang C S, Xiao L, Wang W, et al. An adjustment method for active reflector of large high-frequency antennas considering gain and boresight. Res Astron Astrophys, 2017, 17: 043

15 Haddadi A, Ghorbani A. Distorted reflector antennas: Analysis of radiation pattern and polarization performance. IEEE Trans Antennas Propagat, 2016, 64: 4159-4167

16 Parker D H, Payne J M, Shelton J W, et al. Weadon, instrument for setting radio telescope surfaces. In: Proceedings of ASPE 2000 Annual Meeting. Green Bank, 2000. 21-24

\title{
Preliminary study of regulation technology of wind field distribution on QTT site based on test of equivalent wind field
}

\author{
WANG CongSi ${ }^{1,2 *}$, WANG XueQing ${ }^{1}$, XU Qian $^{2 *}$, WANG Na ${ }^{2}$, ZHENG YuanPeng $^{3}$, \\ DU Biao ${ }^{3}$, LIAN PeiYuan ${ }^{1}, \mathrm{XUE}_{\text {Song }}{ }^{1}$, YAN YueFei ${ }^{1}$, DUAN YuHu ${ }^{4}$, SHI Yu ${ }^{5} \&$ JIA Yu ${ }^{5}$ \\ ${ }^{1}$ Key Laboratory of Electronic Equipment Structure Design, Ministry of Education, Xidian University, Xi'an 710071, China; \\ ${ }^{2}$ XinJiang Astronomical Observatory, Chinese Academy of Sciences, Urumqi 830011, China; \\ ${ }^{3}$ CETC No. 54 Research Institute, Shijiazhuang 050081, China; \\ ${ }^{4}$ CETC No. 39 Research Institute, Xi'an 710065, China; \\ ${ }^{5}$ Department of Mechanical Engineering, University of Chester, Chester CHI 4BJ, UK
}

The effect of wind gust on the large reflector antenna is one of the main factors that can affect the antenna performance and therefore, this effect must be minimized to meet the strict performance requirement in the world largest steerable telescope, which is QiTai Telescope (QTT). In this paper, the characteristics of the topography as well as the wind distribution around QTT site have been analyzed and consequently, a technology for improving the wind distribution in an active way has been proposed. Additionally, an equivalent wind distribution test rig for the proposed technology has been built in the lab and the corresponding experiment has been carried out. The experimental data indicated that the proposed technology was a promising tool for regulating the wind distribution for the large reflector antenna and it was found that the proposed technology can significantly reduce the wind speed as well as the wind impact range after the wind regulation has been given in the test. The results in this paper has provided a solid foundation for the regulation of the wind distribution of the QTT site.

radio telescope, reflector antenna, wind field distribution, wind field regulation

PACS: 95.55.Jz, 84.40.Ba, 92.60.Fm, 89.20.Bb

doi: $10.1360 /$ SSPMA2018-00422 